\title{
Virtual Medium for Design Participation: A Shared Perceptual Understanding in an Urban Design Approach
}

\author{
Shuva Chowdhury ${ }^{1}$ and Marc Aurel Schnabel ${ }^{2}$ \\ 1,2 Victoria University of Wellington, Wellington, New Zealand \\ \{shuva.chowdhury, marcaurel.schnabel\}@vuw.ac.nz
}

\begin{abstract}
Due to lack of communication tools, the non-experts in a participatory urban design process face difficulty to take part actively in the stage of design ideation and generation. Mostly, the design ideas stay in conceptual form and do not provide enough perceptual understanding to conceive the design actions fully. The research hypothesises that an Immersive Virtual Environment (IVE) instrument enhances layperson's urban design participation and collaboration during the early stage of the design generation. The research involves non-expert stakeholders as co-designers for a neighbourhood design in New Zealand. The paper discusses as a parallel reporting with other coming articles on how the IVE instrument facilitates successful design collaboration among fellow laypersons to design their own neighbourhood. A protocol analysis validates the success of design communication happened during non-experts design engagements. An expert evaluation is done to rank the generated design in responding to understand the ideas. In conclusion, the article speculates that an IVE assisted participatory urban design process empowers laypersons to take part actively in urban spatial design.
\end{abstract}

Keywords: Co-design, Design Participation, Urban Design, Immersive Virtual Environment, Laypeople

\section{Introduction}

The quality urban design is that which response sufficiently to social, economic and environmental issues through the physical design. The traditional design approach has not had the tools to visualise urban form concurrently during the decision-making stage. They do not allow non-experts to participate in the early stages of design ideation and generation. The research engages laypersons using a virtual communication system for design discourse, which leads to producing meaningful urban design outcome. These tools offer a ubiquitous virtual interaction platform to produce and visualise iterative design ideas. Engaging laypersons from a neighbourhood in this way able them to create and collaborate on designs, including important perhaps less exciting design elements like driveways and fences. The paper is a part of the continuous report of Immersive Virtual Environment (IVE) engagement of laypersons from a low-density suburb Karori, New Zealand. In our earlier work, Chowdhury and Schnabel (2018) describe a set-up for VR community engagement. The article discusses the design outcome and the nature of design participation happened during the laypersons' engagement. It also reports a survey on an urban design consultation process held by the city council, where the participants stated their interest to take part in the design ideation process. It concludes by comparing the results with conventional consultation methods of urban

Revisiting the Role of Architecture for 'Surviving' Development. 53 ${ }^{\text {rd }}$ International Conference of the Architectural Science Association 2019, Dr. Avlokita Agrawal (ed.), pp. 125-134. (C) 2019 and published by the Architectural Science Association (ANZAScA). 
design and discussing how IVE instrument facilitates co-design communication to generate meaning design outcome.

\section{Design and Design Representation}

A designer uses different representation techniques to communicate with co-workers, clients or user groups (Akin \& Weinel, 1982). Designers always seek suitable means to construct imaginations to express design concepts and turn the concept into visible artefacts (Chan, 2011). It is essential to investigate critically the understanding of virtual 3D artefacts in design representation which allow laypersons to ideate and generate meaningful urban design options.

\subsection{Design as Social Process and Using Artefacts}

Traditional artefacts such as drawings, product samples, models, and now virtual artefacts as 3D models are used to mediate the journey of a building concept to the actual built form. Most often, these artefacts are produced for different purposes and obviously for people with different levels of understanding of the design and construction process. Luck (2007) studies design practice by observing the conversations that surround the use of physical artefacts at the early stages of building design and reveals that the appropriateness of the method of conversation develops users' understanding of the design, as well as the knowledge that is embedded in the artefacts themselves. The design conversation builds the user's confidence in the appearance of the design, rather than only by the ability of the artefacts to represent a future reality. The artefacts embody the current knowledge of the design in its present status, but during a conversation, it prompts discussion of ideas to modify the design. 'The act of interpretation' is acting as part of the design process.

Henri Lefebvre (2003) argues two different design worlds between experts and non-experts. He defines abstract space for the experts' world and concrete space for non-experts world. Experts such as planner, architects and designers create the physical environment in the concrete space by the tool of abstraction and representation. That is why non-experts in concrete space need to conceive design through abstract space. Since modernism, these two worlds were separated, where professionalism always stood apart, and people were treated as the subject of reactive information. A new form of inbetween space can be formed when these two worlds re-join (Lee, 2006), where the realm of design collaboration comes in. Lee develops a diagram to show the three modes of participation (Figure 1). He explains design participation as tactics as the format represent a universal way of design intervention. The design participation mode comes up to empathize the design experience with the inclusion of collaboration between experts and laypersons. It attempts to redefine the concept of 'Design Participation' to investigate creative ways to design with users. Lee (2006) mentions that design practices working in abstract mode with limited contact with users are called Design Participation for innovation and if people are working as a designer for their projects are called Design Participation for motivation. 
Design participation for collaboration happens in between these two realms, which aim to encourage the co-design process.

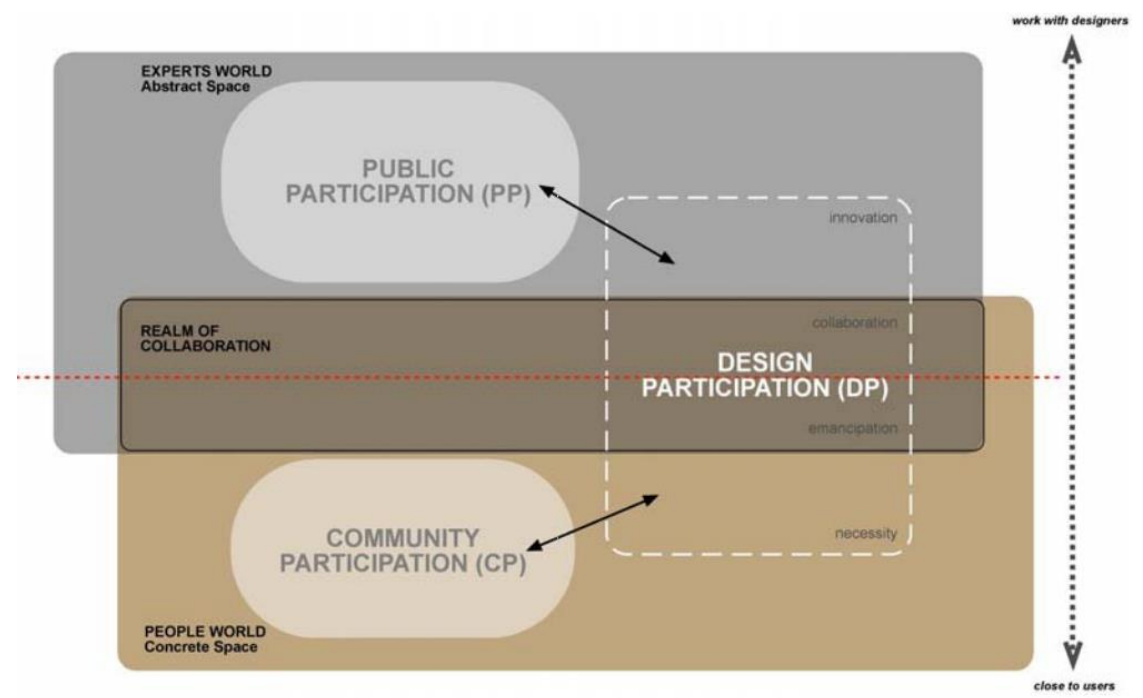

Figure 1 Design, Public and Community Participation in the abstract and concrete space (Lee, 2006).

\section{Participatory Urban Design Process and Tools}

The conventional urban design process includes institutionalised techniques and knowledge of trained professionals who utilise empirical knowledge in dealing and designing an urban situation which only can be understood by the fraternity of those institutions. Conventional urban design techniques cannot offer flexibility to cater to a wide range of social issues in the design process (Kiddle, 2011). Methods like design charrettes and planning workshops already have seen their good days in such democratic engagement in the design processes (Batchelor \& Lewis, 1985; Knevitt \& Wates, 1987; Steinø et al., 2013). However, in spite of having such established methods, there are still differences exist in thinking and communicating language between experts and laypersons (Forester, 1988; Friedman, 1973). The lack of engaging ways eventually pushes researchers to rethink for a new form of design-decision making platform where nonexperts can fully understand the spatial implications of planning and design decisions.

Healey (1997) points out the demands of public participation in decision-making for more accountability on the parts of stakeholders. The integration also encourages social resiliency. It facilitates citizen participation through informing citizen about their rights, responsibility and decision to show their interest in the urban design process. Such participation process also promotes people's attitude to create and manage the urban environment. However, it is hard to exclude the conventional design process, as at the end the professionals have the sole responsibility to the final product. Again, due to the lack of design communication methods, the citizen cannot fully engage in the professional's design process. The existing participatory design process only can allow collective discussion on the planning stage, not in fullscale spatial intervention. A new design communication process requires which can facilitate spatial design collaboration with perceptual understandings between citizen themselves and other stakeholders. 
At first Design Research Society proposed that computer-aided design and human-computer interaction could be potentially combined to develop a system for design participation (Cross, 1972). Based on that concept, now advanced computational tools capable of generating 3D artefacts for seamless design communication which have affordance (Gaver, 1991) for non-experts to involve in design ideation process. These tools also align with the suggestive urban design toolkit of New Zealand (Mfe.govt.nz, 2009). The toolkit is developed for dealing participatory design process in urban development projects to collect consensus from the end-users. They encourage to use interactive visualisation tool to engage people. They are giving more importance of using design consultation techniques, which has limited scope for end-users to communicate design ideation on building shape, form and types with fellow participants. Moreover, the process of design consultation is usually a oneway communication where there is no scope of the participants to generate new design alternatives (Mattelmäki \& Visser, 2011). The article reports a survey on an urban design consultation where the community residents express their interest to take part in the design generation process.

\section{Research Methodology and Reports}

Within a framework of qualitative research, a series of surveys and experiments were set up to investigate the scope of laypersons' active design ideation, generation and collaboration in neighbourhood design. The methodology incorporates a preliminary survey of urban design consultation, developing the VR instrument, engaging laypeople in IVE urban design, a survey on IVE experiment, an audio recording of the design conversation, transcribing recorded data, protocol analysis and expert evaluation (Figure 2). This article reports on the survey of urban design consultation, 3D skill level of the design participants, the protocol analysis of the IVE design communication and expert evaluation. The extended report of the protocol analysis is published in Chowdhury and Schnabel (2019).

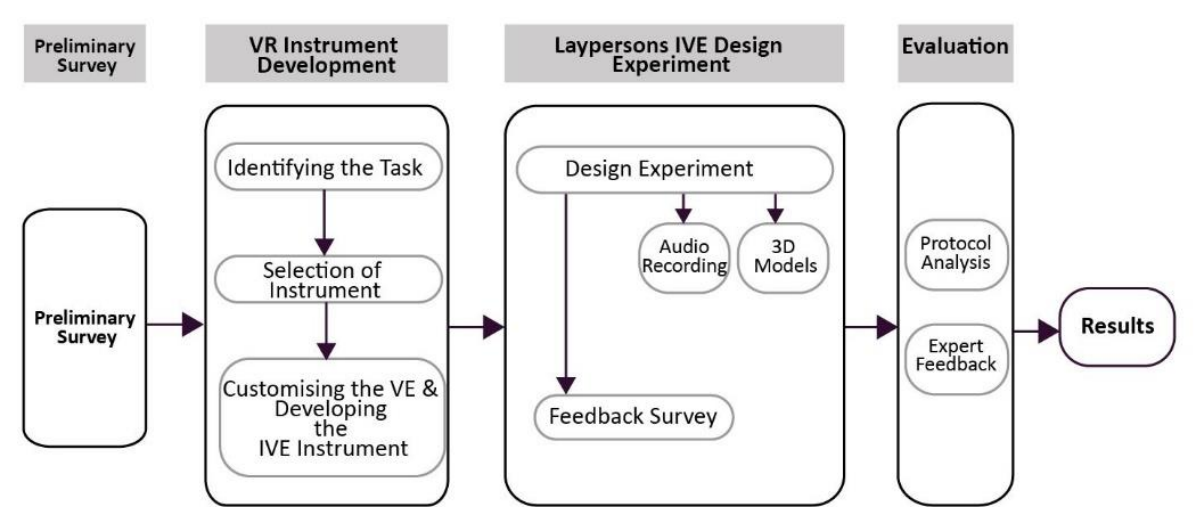

Figure 2 Research Methodology.

\subsection{Preliminary Survey}

Wellington City Council (WCC), ran a design consultation with the Karori Community people on $7^{\text {th }}$ November 2018, where they presented four Karori Neighbourhood design options. The design ideas were presented in a 2D colour printed medium with a perspective and a plan for every design option. The session started with a presentation, then question answering session with the Karori people and finally a 
voting session on the design options. The preliminary survey aims to identify the task to involve laypersons' in IVE design participation. Survey results come to the conclusion that the participants want to take part as active designers in the design process. There are 30 participants. The measuring value of each answer is the level of choices like 'Absolute'; 'It is Ok', 'Neutral', 'Not Really'and 'Not at all'.

In terms of designing their own design, almost $53.8 \%$ of participants want to have own design along with proposed set design solution (Figure 3). There are $34.6 \%$ of people who also want to have a set design solution. There are also a handful of people who completely want to design by themselves. The results indicate that the community people want to take part in the urban design ideation and generation stage with more understanding of the proposed spatial context.

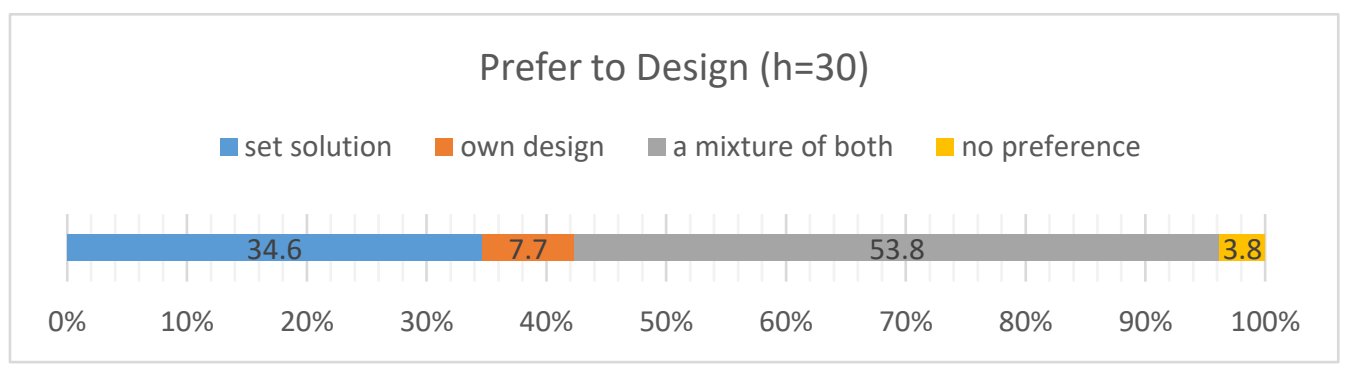

Figure 3 Prefer to design.

\subsection{IVE Experiment}

The IVE experiment method is akin to Schnabel's (2011) immersive virtual environment design studio research. A unit diagram is shown in Figure 4, where Designer A designs in IVE and Designer B communicate with the design representation through a 2D display screen. Supporting Kocaturk and Codinhoto (2009) definition, here, the IVE designer interacts with digital media as internal representation to mentally construct, generate and model the design artefact. Simultaneously, Designer A generates external representation to communicate design information with Designer B (or other stakeholders) through the output in the 2D display screen. Eventually, it results collective creativity. The technique has advantages by allowing designers to tackle real-world issues through a discursive process and create a perceptual understanding of the generated ideas in the VE. 


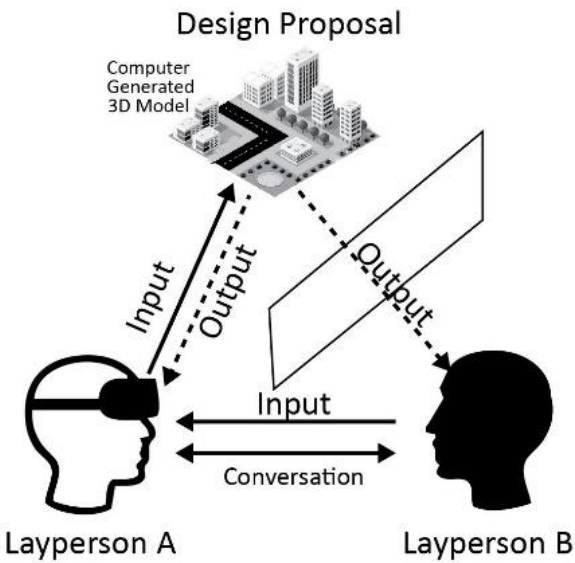

Figure 4 One unit of 'Enhanced Communication”.

\subsection{Meaningful Design Outcome}

The IVE design experiment task is described in our earlier paper Chowdhury and Schnabel (2018). While the designers are designing, the visual information helps them to speculate different functional arrangement for the site. Designers propose mixed-use commercial spaces with restaurants/parks/ play centers/ art pavilions/ a place for food truck standings. We report eight design alternatives. The produced design options are:

Option 1: Mixed-use urban park

Option 2: Outdoor café and event space

Option 3: Mixed-use urban park

Option 4: Playground

Option 5: Night-market, food stall, community event centre

Option 6: Mixed-use retail and café

Option 7: Play centre

Option 8: Urban park

Figure 5 shows except design Options 5 and 7, rest of the design teams have at least one 3D skilled participant. However, all of them able to produce design alternatives due to the IVE experimental setup of design collaboration. 


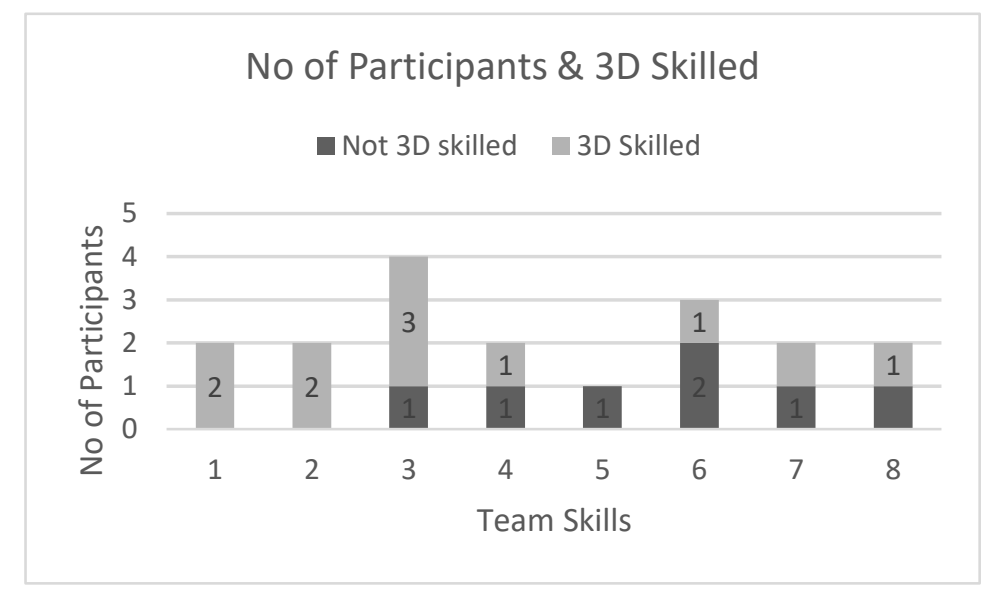

Figure 5 No of Participants and 3D skilled in produced design options.

\subsection{Design Communication and Participation}

The recorded conversation is transcribed to analysis the design conversation through protocol analysis. Based on Tsai et al. (2009), a coding scheme was developed to evaluate virtual design communication and collaboration. The four major categories of the scheme are 1. Communication control, 2. Design communication, 3. Social communication and 4. Communication technology. The result shows that there was evidence of successful design communication happened between the participants (Figure 6). Around $62.37 \%, 62.79 \%$ and $46.51 \%$ of the conversation respectively for option 2,4 and 7 happen about deriving design concept. It indicates that the VR engagement facilitated the scope of collaborative design ideation. Similarly, there is frequent non-task-related social communication happened, which also proves the presence of social communication (Figure 7 ). Almost $75.76 \%, 16.67 \%$ and $80.52 \%$ of the conversation

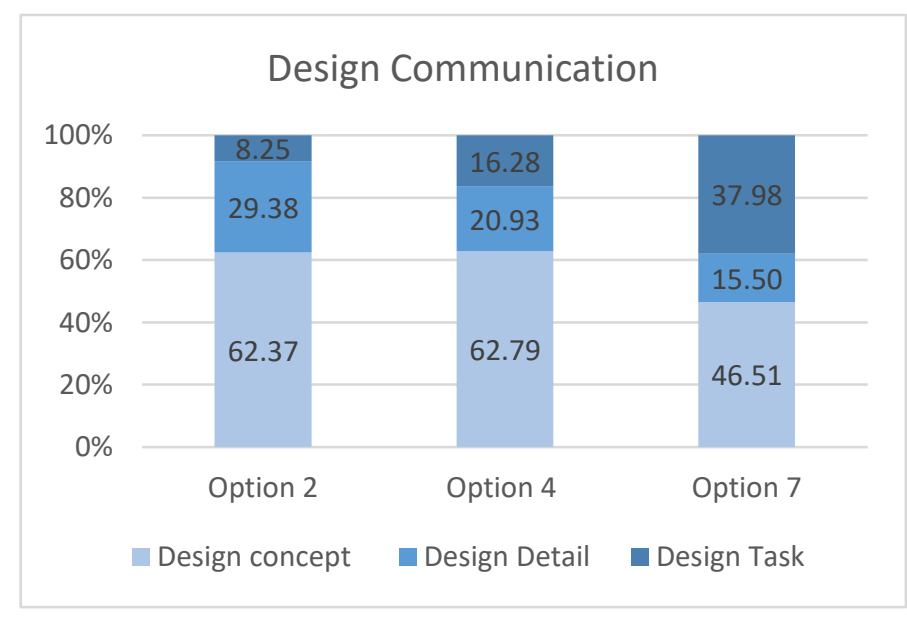

Figure 6 Design Communication. 
respectively for option 2, 4 and 7 occur as non-task related social communication. It indicates the design actions generated by Designer $A$ is communicated to Designer $B$ in a successful way to form a meaningful outcome.

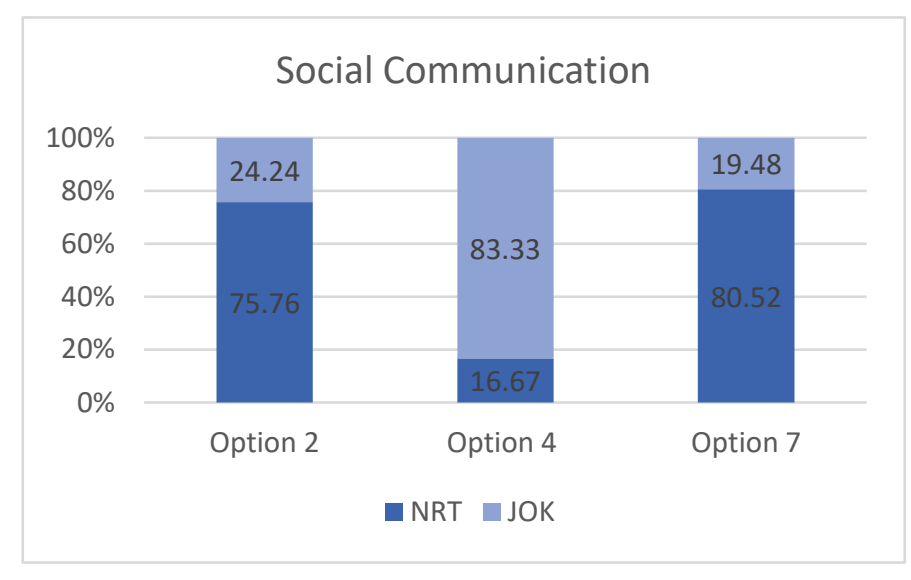

Figure 7 Social Communication.

\subsection{Expert Evaluation}

Two architects have evaluated the produced design ideas. The evaluation criteria had been set on functionality, aesthetics and experiential qualities of the design options. Design options 2 and 4 got the highest average points (Figure 8). In general, evaluating aesthetics in design is mainly a matter of grasping its sensuous qualities. Assessing aesthetic qualities it does not mean that it exhausts all the different properties that design compasses, like functionality and sustainability. The evaluation on aesthetic reflects how the laypeople's proposed building form and its function have been communicated to the experts.

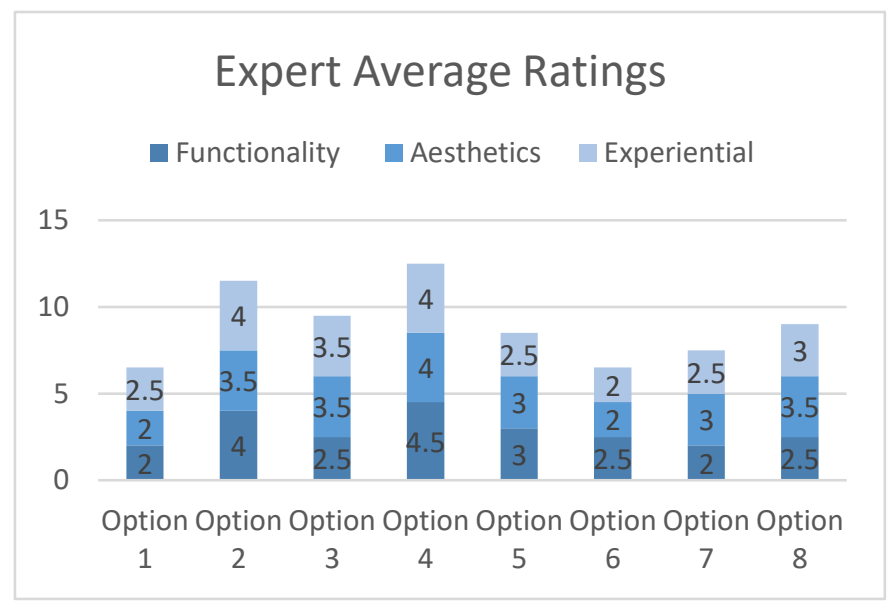

Figure 7 Average Ratings Attributes $1=$ Not at All, 2= Not Really, 3=Neutral, 4=It is OK, 5=Very Much. 


\section{Discussion}

Through engaging non-experts, the research impacts on the perceptual affordance created through the interaction of virtual 3D models, immersive, non-immersive visual information and verbal conversation. Participants actively take part in the design ideation and generation stage. Only one person was allowed to design in the immersive world, and others were allowed to provide verbal feedback. In between their conversation, the computer produced 3D urban forms which provide visual feedback to continue the discourse. In contrast to Lefebvre, here the non-experts are designing in the abstract space, where they usually participate in concrete space. That means the VR instrument is facilitating design participation, which is re-joining the scope of non-expert's role to the expert's role. The report of the protocol analysis also shows that design communication happened regarding task-related design actions. It means the participants took design decision through their action in the IVE environment. Moreover, the experts' evaluation legitimizes the design outcomes as products of meaningful design participation.

Conventionally participatory design activities are dealt with urban professionals in a media either resembles paper and pencil method or models or most recently in a non-immersive game-based virtual environment. On the contrary, our research shows that the end-users are getting the opportunity to involve in the discussion by being perceptually inhabited in the urban context. The IVE instrument and the design engagement set-up helped the laypeople to decide on specific perceptual qualitative parts of urban form through collaboration. The procedure of design engagement helps to facilitate design discourse through visual and verbal interaction. The design engagement empowers the participants to design building form, shape, texture and also placement in the real context. So, from the perspective of the enduser, they are acting as a designer in the urban design process, and the expert plays the role of facilitators. It seems the roles of designers and users are coming to a level where the users can create and propose design ideas through collaboration with fellow designers. The participatory mindset values people as cocreator in the design process. This immersive virtual participatory design is bridging the power between designers and users, where the users are getting the privilege to put their design interest collaboratively by being perceptually inhabited in the urban context.

\section{Conclusion}

The research undertaken has not only moved the IVE from architecture design to participatory urban design but also from the expert realm to non-expert realm in a new, unforeseen and materially different way. It moves us closer towards a better understanding of integrating an easy intuitive IVE interface for laypeople's spontaneous design participation. It demonstrates that integrating suitable IVE instrument minimizes the distance between the non-expert's collective design imagination and its representation during the initial stage of urban design. Most tools in IVE are used for design consultation through presentation or simulation. Designing within IVE leverages local people to visualize and discover their design concepts together for their community. It empowers laypersons to express, explore and convey their imagination to engage actively in urban design. By analysis of design conversation and representations, the research has demonstrated that laypeople's collaborative conception, perception and understanding of spatial volumes within IVE contribute to the quality, understanding and designing urban space and forms. Moreover, its easy nature of design creation, communication and collaboration, offers an opportunity for experts and non-experts collaboration in the early stage of urban design. Therefore, it re-establishes a new direction to include stakeholders in the design process where the 
intuitive design communication empowers non-experts to participate in a spatial discussion on designing future neighbourhood.

\section{Acknowledgements}

The research reported in this paper has been supported by New Zealand's National Science Challenge Building Better Homes, Towns and Cities (BBHTC) - Shaping Places: Future Neighbourhoods. It has been approved by the Victoria University of Wellington Human Ethics Committee 0000025705.

\section{Reference}

Akin, O., \& Weinel, E. F. (1982). Representation and architecture.

Batchelor, P., \& Lewis, D. (1985). Urban Design in Action: The History, Theory and Development of the AIA's Regional/Urban Design Assistance Teams Program (RUDAT). School of Design, North Carolina State UniversityRaleigh and American Institute of Architects, 29.

Chan, C.-S. (2011). Design representation and perception in virtual environments. In Collaborative Design in Virtual Environments (pp. 29-40): Springer.

Chowdhury, S., \& Schnabel, M. A. (2018). Communication and Participation in Virtual Environments. Paper presented at the International Conference of the Architectural Science Association.

Chowdhury, S., \& Schnabel, M. A. (2019). Laypeople's Collaborative Immersive Virtual Reality Design Discourse in Neighborhood Design. Frontiers in Robotics and Al, 6, 97.

Cross, N. (1972). Design participation: Academy Editions.

Forester, J. (1988). Planning in the Face of Power: Univ of California Press.

Friedman, J. (1973). Retracking America: A Theory of Societal Planning. In: New York: Doubleday.

Gaver, W. W. (1991). Technology affordances. Paper presented at the Proceedings of the SIGCHI conference on Human factors in computing systems.

Healey, P. (1997). Collaborative planning: Shaping places in fragmented societies: UBC Press.

Kiddle, R. (2011). Learning outside the box: designing social learning space. Oxford Brookes University,

Knevitt, C., \& Wates, N. (1987). Community architecture. In: London: Penguin.

Kocaturk, T., \& Codinhoto, R. (2009). Dynamic coordination of distributed intelligence in design. Paper presented at the the Proceedings of the 27th eCAADe Conference, Computation: The New Realm of Architectural Design, Istanbul (Turkey).

Lee, Y. (2006). Design Participation Tactics: Redefining User Participation in Design. Paper presented at the Design research society international conference, Lisbon.

Lefebvre, H. (2003). The urban revolution: $U$ of Minnesota Press.

Luck, R. (2007). Using artefacts to mediate understanding in design conversations. Building Research \& Information, 35(1), 28-41.

Mattelmäki, T., \& Visser, F. S. (2011). Lost in co-x. Proceedings of the IASDR2011.

Mfe.govt.nz. (2009). Urban design toolkit: Third edition. Retrieved from https://www.mfe.govt.nz/sites/default/files/urban-design-toolkit-third-edition.pdf

Schnabel, M. A. (2011). The immersive virtual environment design studio. Collaborative Design in Virtual Environments, 177-191.

Stein $\varnothing$, N., Benbih, K., \& Obeling, E. (2013). Using Parametrics to Facilitate Collaborative Urban Design: An Attempt to Overcome some Inherent Dilemmas. Planum, The Journal of Urbanism, 26(1), 13.

Tsai, J. J.-h., Wang, X., \& Huang, Y. (2009). Protocol Analysis of Collaborative Designs of Different Scales in Real and Virtual Environments. In N. Gu, M. J. Ostwald, \& A. Williams (Eds.), Computing, Cognition and Education: Recent Research in the Architectural Sciences (pp. 233-245). Australia: ANZAScA. 